\title{
Large Power Transformer Fault Diagnosis and Prognostic Based on DBNC and D-S Evidence Theory
}

\author{
Gang Li', Changhai Yu'1, Hui Fan², Shuguo Gao², Yu Song'1, Yunpeng Liu ${ }^{3}$ \\ ${ }^{1}$ School of Control and Computer Engineering, North China Electric Power University, Baoding, China \\ ${ }^{2}$ State Grid Hebei Electric Power Research Institute, Shijiazhuang, China \\ ${ }^{3}$ School of Electrical and Electronic Engineering, North China Electric Power University, Baoding, China \\ Email: 15031221890@139.com,15830105382@139.com, liuyunpeng@ncepu.edu.cn, mdjych@foxmail.com, \\ ququ_er2003@126.com,syu1999@163.com
}

How to cite this paper: Li, G., Yu, C.H., Fan, H., Gao, S.G., Song, Y. and Liu, Y.P. (2017) Large Power Transformer Fault Diagnosis and Prognostic Based on DBNC and D-S Evidence Theory. Energy and Power Engineering, 9, 232-239.

https://doi.org/10.4236/epe.2017.94B028

Received: February 13, 2017

Accepted: March 30, 2017

Published: April 6, 2017

\begin{abstract}
Power transformer is a core equipment of power system, which undertakes the important functions of power transmission and transformation, and its safe and stable operation has great significance to the normal operation of the whole power system. Due to the complex structure of the transformer, the use of single information for condition-based maintenance (CBM) has certain limitations, with the help of advanced sensor monitoring and information fusion technology, multi-source information is applied to the prognostic and health management (PHM) of power transformer, which is an important way to realize the CBM of power transformer. This paper presents a method which combine deep belief network classifier (DBNC) and D-S evidence theory, and it is applied to the PHM of the large power transformer. The experimental results show that the proposed method has a high correct rate of fault diagnosis for the power transformer with a large number of multi-source data.
\end{abstract}

\section{Keywords}

Power Transformer, Prognostic and Health Management (PHM), Deep Belief Network Classifier (DBNC), D-S Evidence Theory

\section{Introduction}

Power transformer is the important equipment in power system which has complex internal structure and various fault types [1]. At present, most of the power transformer's PHM methods are based on a certain factor or a number of factors to make judgments [2], it does not take into account the overall operating condi- 
tions of the transformer, defect information, maintenance history, family history, and other comprehensive state information, due to the limitations of the test methods, the imprecision of knowledge and other reasons. Therefore, the information appears the fuzzy and random characteristic, and then the exact description of the operation and maintenance department for internal coupling interaction and the fault evolution of transformer are not enough. For the uncertainties of power transformer's PHM, accuracy and timeliness of fault diagnosis, prognostic and health management results are far from practical requirements.

Multi-source information fusion technology is a new information processing technology developed in recent years [3]. It makes full use of multiple sensor resources, and combines the complementary and redundant information of various sensors in space and time based on some optimization criterion, in order to achieve the best synergistic effect, increase the survivability of the system, expand the spatial and temporal coverage, improve the reliability of the results, reduces the ambiguity of information. The multi-source information fusion technology applied in transformer's PHM, can make up the disadvantages of the single data source in the traditional PHM method. It can analyze the potential information from a large amount of complex transformer characteristic data accurately and efficiently. So as to determine the transformer condition of and predict the transformer fault, reduce the harm caused by the transformer fault, and ensure the safe and stable operation of the power system [4] [5] [6].

\section{PHM and DBN Theory}

\subsection{Primary Mission of PHM}

PHM aims to extend the life cycle of engineering equipment while reducing the cost of development and maintenance [7]. There are three main parts in the whole cycle of power transformer's PHM, which are fault diagnosis, fault prognostic and condition-based maintenance [8]. The purpose of fault diagnosis is to diagnose and identify the root causes of transformer failure; the root causes can provide useful information for the prognostic models as well as feedback for transformer design improvement. Prognostic takes the processed data as well as the existing system model or failure mode analysis as inputs, and then use the prognosis algorithm to online update the degradation models and predict failure times of the power transformer. CBM is the use of prognostic outcomes, considering the costs and benefits of different maintenance operations to determine when and how to perform preventive maintenance to minimize operating costs and risks.

Above these three tasks need to be executed dynamically and in real time, this paper presents a new method for fault diagnosis of power transformer. The research scheme of large power transformer's PHM, as shown in Figure 1.

\subsection{Deep Belief Network}

Deep Belief Network (DBN) is a kind of deep learning method, has a strong ability to extract features from a large number of samples in order to better classify, and improve the accuracy of classification. The method has been successfully 


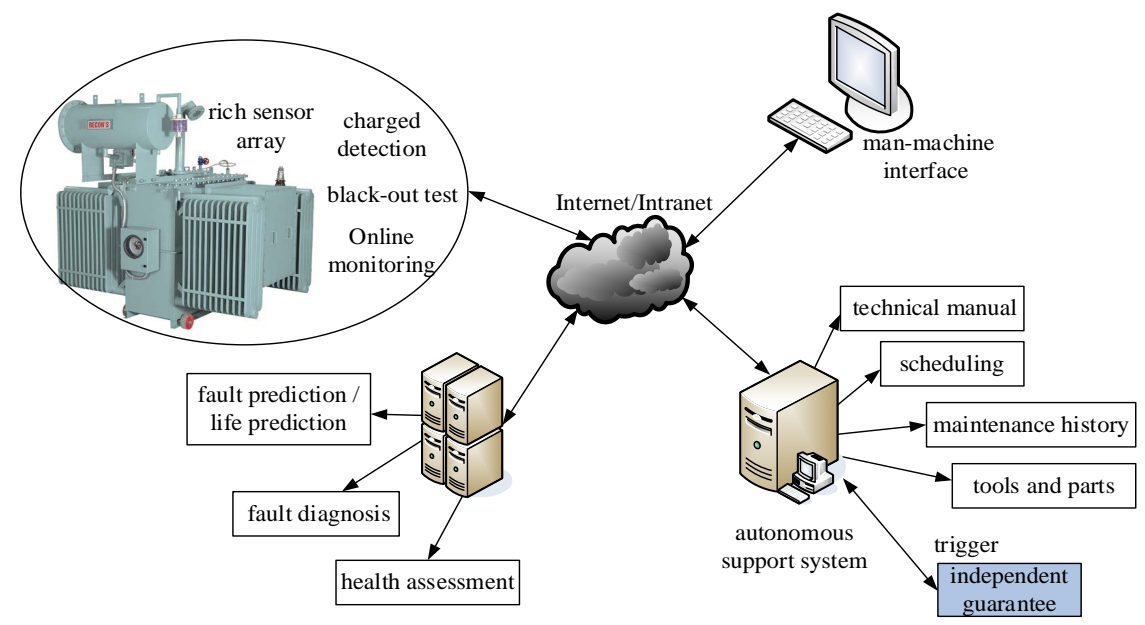

Figure 1. Power transformer's PHM scheme.

applied to the classification problem, and shows some advantages, is a hotspot of current international research on machine learning [9].

DBN was proposed by Professor Geoffrey Hinton in 2006 [10], which is a probabilistic generative model to establish a joint probability distribution between observed data and labels, evaluates both $P$ (Observation|Label) and $P$ (Label|Observation). The structure, which is composed of a plurality of Restricted Boltzmann Machines (RBM) stacked, uses layer by layer training methods. It solves the training problem that the traditional Neural Network (NN) training method is not suitable for multi-layer network, the DBN training is divided into two stages: pre-training and tuning.

\subsubsection{Pre-Training}

Pre-training is the process essentially which is the initialization of the network parameters, uses layer by layer unsupervised feature optimization algorithm. Initialized network parameter is the connection weights between the layers and the offset value of each layer neurons. As an example to introduce the hierarchical structure of RBM, as shown in Figure 2.

RBM contains a visible layer $v$ and a hidden layer $h$, there is no connection between each layer units, full connection between layers. Assume that the $v$ layer has $n$ visible units, $h$ layer has $m$ hidden units. So, RBM as system energy is defined as Equation (1):

$$
E(v, h \mid \theta)=-\sum_{i=1}^{n} a_{i} v_{i}-\sum_{j=1}^{m} b_{j} h_{j}-\sum_{i=1}^{n} \sum_{j=1}^{m} v_{i} W_{i j} h_{j}
$$

where $v_{i}$ is the condition of the first $i$ visible unit, $h_{j}$ is the condition of the first $j$ hidden unit, $\theta=\left\{W_{i j}, a_{i}, b_{j}\right\}$ is the RBM parameter, $W_{i j}$ is the connection weight between visible unit $i$ and hidden unit $j, a_{i}$ is the offset of visible unit $i, b_{j}$ is the offset of hidden unit $j$. Based on the energy function, the joint probability distribution of $(v, h)$ can be obtained by Equation (2):

$$
P(v, h \mid \theta)=\frac{e^{-E(v, h \theta)}}{Z(\theta)}
$$




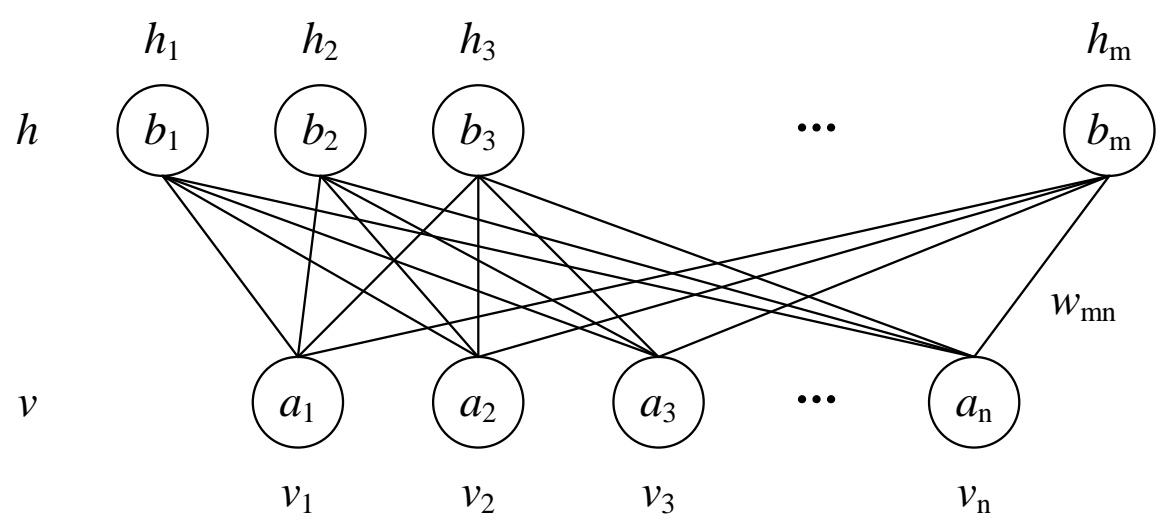

Figure 2. A hierarchical structure of RBM.

where $Z(\theta)=\sum_{v, h} e^{-E(v, h \theta)}$ is the normalization factor, i.e. partition function. The marginal distribution (Also known as likelihood function) of the joint probability distributions $P(v, h \mid \theta)$ can be expressed as Equation (3):

$$
P(v \mid \theta)=\frac{1}{Z(\theta)} \sum_{h} e^{-E(v, h \mid \theta)}
$$

\subsubsection{Tuning}

After the pre-training is completed, each layer of RBM can get the initialization parameters, form the preliminary framework of DBN, then we need tune training for DBN, further optimize the parameters of each network layer, in order to make the network discrimination performance better. The tuning process is supervised learning process, namely using unlabeled data for training, then use the $\mathrm{BP}$ algorithm fine tuning the network parameters, finally to achieve the global optimal network. The performance will be better than the effect of BP algorithm training, because it only needs a local search for the network parameter space, compared to BP neural network, it has fast training speed, and short convergence time.

\section{Multi-Source Information Fusion Model of Power Transformer's PHM}

The multi-source information fusion involves many aspects of theory and technology, including signal processing, estimation theory, and fuzzy theory, clustering analysis, neural network and artificial intelligence and so on. Information fusion can be divided into 3 levels, including data fusion, feature fusion and decision fusion. The main methods used are Bayesian inference, D-S evidence theory, fuzzy theory, expert system and so on.

D-S evidence theory was put forward by Dempster in 1967 [11], then expanded and developed by Shafer, so the evidence theory is also called D-S evidence theory. D-S evidence theory has been widely used in multi-sensor information fusion. In the evidence theory, in order to describe and deal with the uncertainty, the concepts of probability distribution function, belief function and likelihood function are introduced. 
1) Probability Distribution Function

Set $D$ as sample space, the propositions in the field are represented by a subset of $D$; the probability distribution function is defined as follows.

Set function $M: 2^{D} \rightarrow[0,1]$, and satisfies $M(\varnothing) \neq 0, \sum_{A \subseteq D} M(A)=1, M$ is called the probability distribution function on $2^{D}, M(A)$ as the basic probability function of $A$.

2) Belief Function and Likelihood Function

Belief function is represented by Bel, Bel function also called lower limit function, let $\operatorname{Bel}(A)$ denote the degree of belief that proposition $A$ is true. Likelihood function is represented by $P I s, \operatorname{Pls}(A)$ denote the degree of belief that not deny $A$. $\lfloor\operatorname{Bel}(A), \operatorname{Pls}(A)\rfloor$ called the trust interval of $A$.

3) Orthogonal Sum of Probability Distribution Functions

When two or more different probability distribution functions are obtained for the same evidence, it is necessary to combine them, i.e. orthogonal sum of probability distribution functions. Let $M_{1}, M_{2}, \cdots, M_{n}$ be $\mathrm{n}$ probability distribution function, its orthogonal sum $M=M_{1} \oplus M_{2} \oplus L \oplus M_{n}$ is Equation (4):

$$
M(A)=K^{-1} \sum_{\cap A_{i}=A} \prod_{1 \leq i \leq n} M_{i}\left(A_{i}\right)
$$

where $K=1-\sum_{\cap A_{i}=\varnothing} \prod_{1 \leq i \leq n} M_{i}\left(A_{i}\right)$.

If $K \neq 0$, then orthogonal sum $M$ is a probability distribution function. If $K=0$, there is no orthogonal sum, said $M_{1}$ and $M_{2}$ contradictions.

According to the general framework of information fusion and the characteristics of the transformer fault, DBN is combined with information fusion and applied to fault diagnosis in this paper. DBN diagnosis belongs to the process of the feature level input and the decision level output in the information fusion sense, and the D-S evidence theory fuse and reasoning the various evidence body of the same framework and come to a unified decision, belongs to the process of the decision level input and the decision level output. The combination can greatly improve the reliability and accuracy of diagnosis. Therefore, this paper established the power transformer multi-fault information hierarchical decision fusion diagnostic model based on the combination of DBN and D-S evidence theory, as shown in the Figure 3. DGA's parameters including $\mathrm{H}_{2} . \mathrm{CH}_{4}, \mathrm{C}_{2} \mathrm{H}_{6}$ and so on, electrical test data including winding unbalanced coefficient, winding dielectric loss, core grounding current.

\section{PHM Example}

This paper using the deep belief network classifier (DBNC) model (as shown in Figure 4 is the DGA gas DBNC model) constructed by [9] is used to classify the sample data. The input of the model is the seven characteristic gas content values (after the standardized treatment) of oil chromatogram on-line monitoring. Finally, the output of the top Softmax classifier is the probability that the corresponding samples belong to different states respectively; the state of maximum probability is the result of classification. Finally, the D-S evidence theory is used 


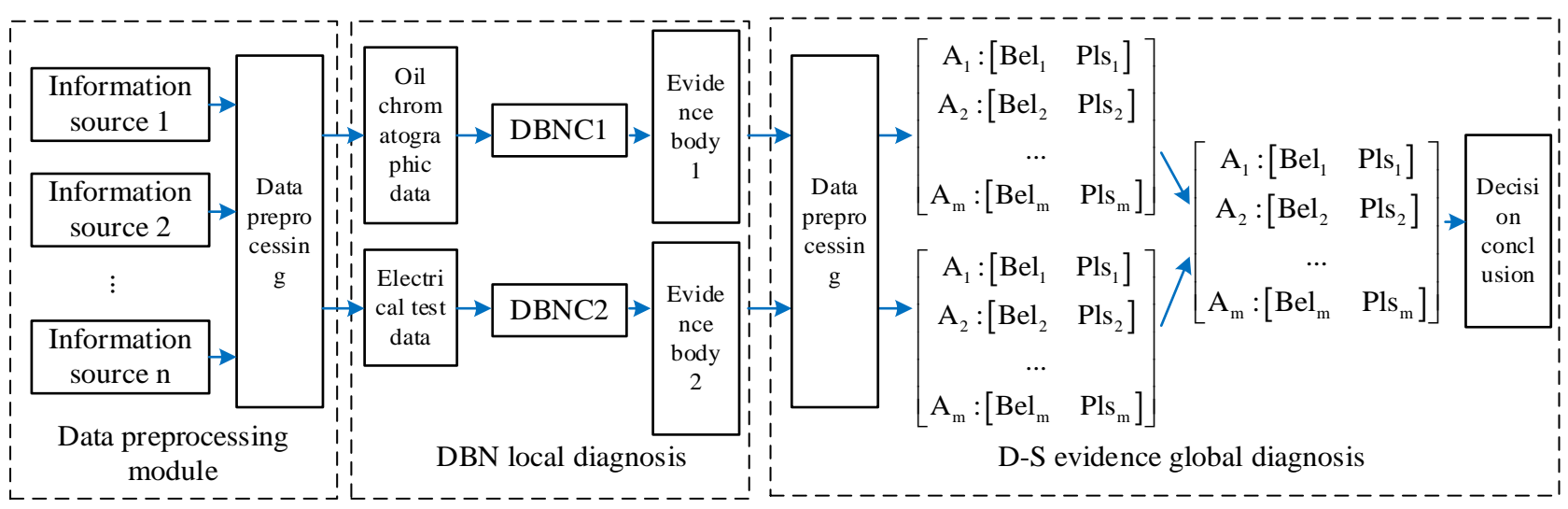

Figure 3. Hybrid diagnostic model of power transformer based on the combination of DBN and D-S evidence theory (multi-source information).

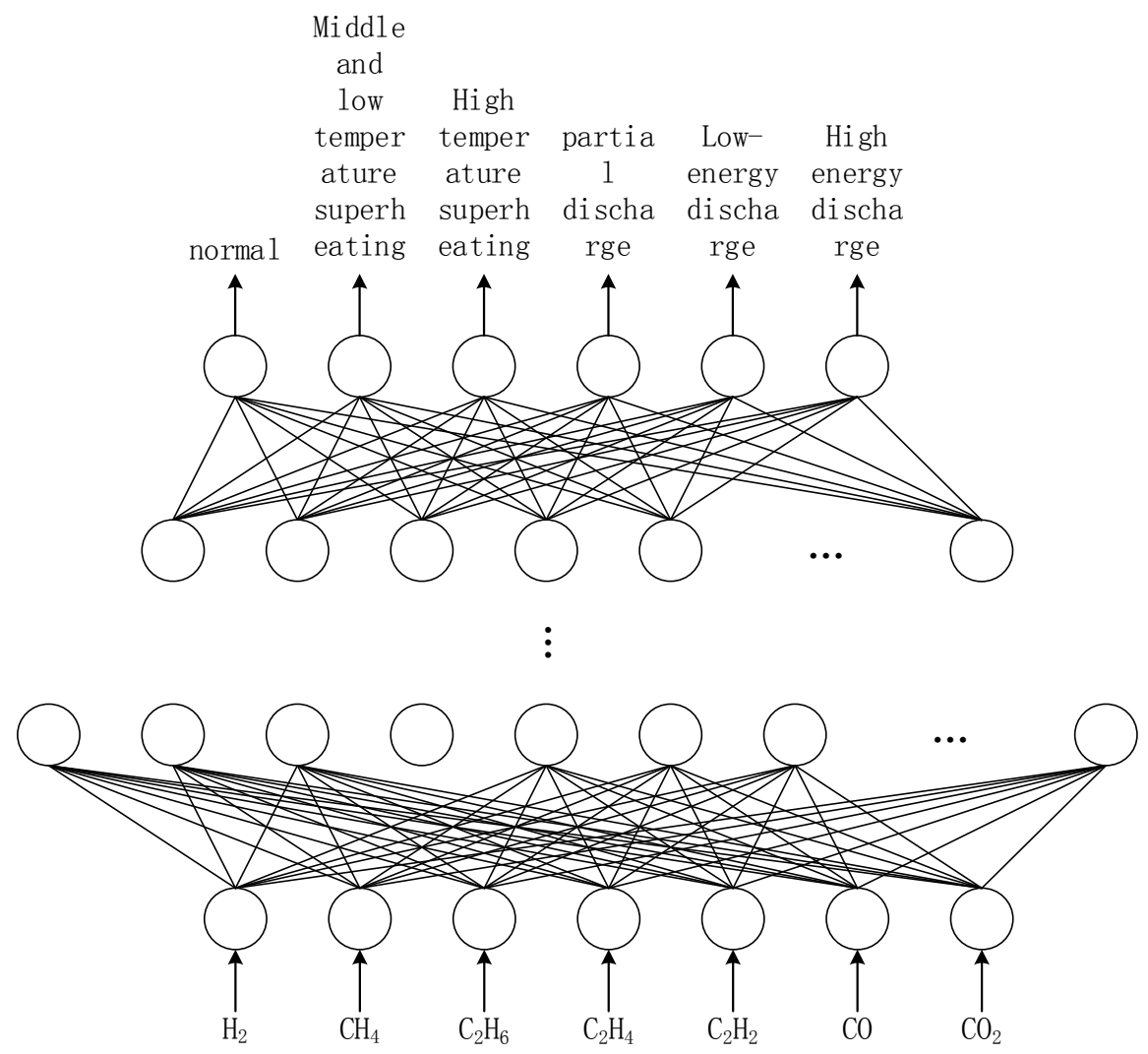

Figure 4. Transformer fault classification model based on DBNC.

to fuse the diagnosis results to get the final result.

This paper collected 1500 sample data of a transformer, the oil chromatogram data are shown in Table 1 . In the electrical test project, the core insulation resistance, winding DC resistance unbalance coefficient, core grounding current exceeds the notice value and other electrical test items are normal. Wire-wound resistor only $65 \mathrm{M} \Omega$ (the notice value is $1000 \mathrm{M} \Omega$ ), winding DC resistance unbalance coefficient is $2.95 \%$ (the notice value is $2 \%$ ), core grounding current is $0.13 \mathrm{~A}$ (the notice value is $0.1 \mathrm{~A}$ ). 
Table 1. The fault transformer oil chromatographic data.

\begin{tabular}{ccccccc}
\hline \multicolumn{7}{c}{ Gas volume fraction/10-6 } \\
\hline $\mathrm{H}_{2}$ & $\mathrm{CH}_{4}$ & $\mathrm{C}_{2} \mathrm{H}_{6}$ & $\mathrm{C}_{2} \mathrm{H}_{4}$ & $\mathrm{C}_{2} \mathrm{H}_{2}$ & $\mathrm{CO}$ & $\mathrm{CO}_{2}$ \\
\hline 64.26 & 84.05 & 23.07 & 148.12 & 1.42 & 827.32 & 4661.26 \\
25.58 & 89.55 & 20.02 & 134.98 & 0.89 & 718.45 & 4231.54 \\
35.13 & 91.48 & 21.98 & 141.54 & 0.93 & 736.54 & 4195.87 \\
28.35 & 91.25 & 19.96 & 139.95 & 0.75 & 781.25 & 4325.28 \\
33.25 & 91.84 & 21.21 & 140.00 & 0.82 & 800.23 & 4521.78 \\
36.03 & 98.75 & 22.64 & 153.01 & 0.89 & 765.74 & 4124.76 \\
\hline
\end{tabular}

Using DBNC classifier to classify the sample data, the diagnosis result accuracy of oil chromatogram data reached $81.53 \%$, the diagnosis result accuracy of electrical test data reached $78.83 \%$. Fusing diagnosis results by D-S evidence theory, the diagnosis accuracy reached $88.56 \%$. We can see the diagnosis results of multi-source information fusion model for fault diagnosis accuracy are higher than the diagnostic results for single or less information sources.

\section{Conclusion}

This paper attempts to introduce the concept of PHM into the field of power transformer, in order to provide a complete reference system for conditionbased maintenance of power transformer. On this basis, a hybrid diagnostic model is proposed in this paper for the fault diagnosis stage of power transformer's PHM cycle, which is based on the deep belief network classifier and D-S evidence theory. The experimental results show that the diagnosis results of the diagnostic model is superior to the single source information; the effectiveness of multi-source information fusion in improving the accuracy of power transformer's fault diagnosis is verified.

\section{Acknowledgements}

This work is supported by National Natural Science Foundation of China (51407076), the Natural Science Foundation of Hebei Province (F2014502050) and the Fundamental Research Funds for the Central Universities (2015ZD28).

\section{References}

[1] Wu, K., Kang, J.S. and Chi, K. (2016) Fault Diagnosis Method of Power Transformers Using Improved Multi-class Classification Algorithm and Relevance Vector Machine.High Voltage Engineering, 42, 3011-3017.

[2] Zhao, W.Q. and ZhuY.L. (2007) Summary of condition assessment for Power Transformer. Transformer, 44, 9-12.

[3] Cui Y., Ma, H. and Saha, T. (2016) Multi-source information fusion for power transformer condition assessment. Power and Energy Society General Meeting. Boston, 17-21, July 2016, 1-5.

[4] Gao, Z.X., Guo, C.G. and Yu, B. (2011) Study of A Fault Diagnosis Approach for Power Grid with Information Fusion Based on Multi-Data Resources. Power System Protection and Control, 39, 17-23. 
[5] Dai, J. F. (2010) On-Line Fault Detection for Transformers Based on Information Fusion. Electrotechnics Electric, 51-55.

[6] Li, Y.W., Li, W. and Han, X.D. (2009) Application of Multi-Sensor Information Fusion Technology in the Power Transformer Fault Diagnosis. International Conference on Machine Learning and Cybernetics. Baoding, 12-15, July 2009, 29-33.

[7] Javed, K. (2014) ARobust And Reliable Data-Driven Prognostics Approach Based on Extreme Learning Machine and Fuzzy Clustering. Université de Franche-Comté.

[8] Tsui, K.L., Chen, N. and Zhou, Q. (2015) Prognostics and Health Management: A Review on Data Driven Approaches. Mathematical Problems in Engineering, 1-17. https://doi.org/10.1155/2015/793161

[9] Shi, X., Zhu, Y.L. and Sa, C. (2016) Power Transformer Fault Classifying Model Based on Deep Belief Network. Power System Protection and Control, 44, 71-76.

[10] Hinton, G.E., Osindero, S. and The, Y.W. (2006)A Fast Learning Algorithm for Deep Belief Nets. Neural computation, 18, 1527-1554. https://doi.org/10.1162/neco.2006.18.7.1527

[11] Dempster, A. P. (1967) Upper and Lower Probabilities Induced by a Multivalued Mapping. The Annals of Mathematical Statistics,38, 325-339. https://doi.org/10.1214/aoms/1177698950

Submit or recommend next manuscript to SCIRP and we will provide best service for you:

Accepting pre-submission inquiries through Email, Facebook, LinkedIn, Twitter, etc. A wide selection of journals (inclusive of 9 subjects, more than 200 journals)

Providing 24-hour high-quality service

User-friendly online submission system

Fair and swift peer-review system

Efficient typesetting and proofreading procedure

Display of the result of downloads and visits, as well as the number of cited articles Maximum dissemination of your research work

Submit your manuscript at: http://papersubmission.scirp.org/

Or contact epe@scirp.org 